\title{
Reducing Unplanned Extubations in the NICU Using Lean Methodology
}

\author{
Bonnie M Powell RRT-NPS, Edeltraud Gilbert MBOE LSSBB, and \\ Teresa A Volsko MBA MHHS RRT CMTE FAARC
}

\begin{abstract}
BACKGROUND: Unplanned extubations can lead to iatrogenic injury and have the potential to contribute to serious safety events. We adopted lean methodology to reduce the unplanned extubation rate in a Level 3b NICU. We hypothesized that the use of a rapid-cycle PDSA (plan, do, study, act) initiative would reduce the unplanned extubation rate. METHODS: Baseline unplanned extubation data were collected from November 1, 2012 to June 6, 2014. A voice of the customer survey ascertained perceptions regarding unplanned extubation causes and impact on care. The confidential survey contained 2 open-ended and 4 closed-ended questions and was distributed to a random sample of nurses and respiratory therapists. A fishbone diagram helped to identify opportunities. Six improvements were identified and rolled out in 2 phases using didactic and kinesthetic techniques. Phase 1 standardized the process for turning intubated infants, assessing endotracheal tube (ETT) placement with growth, and communicating tube position with caregivers. Phase 2 addressed respiratory plans of care, correcting ETT migration, establishing ETT re-securement methods, and standardizing position during radiography. The Fisher exact test was used to determine differences in the number of unplanned extubations per 100 intubated days. Descriptive statistics were used to report survey results. Statistical significance was established at $P<.05$. RESULTS: A $68 \%$ (17 of 25$)$ survey response rate was realized. Baseline data revealed 3.8 unplanned extubations/100 intubated days, and 2.7 unplanned extubations/100 intubated days occurred in the post-improvement phase $(P=.01)$. We noted a statistically significant decrease in the number of intubated days between the pre- and post-improvement groups $(P<.001)$. CONCLUSIONS: Staff underestimated the prevalence of unplanned extubations but recognized the need for improvement. Rapid cycle PDSA significantly reduced the unplanned extubation rate. The decrease in intubated days may have been a by-product of the post-improvement phase improvements, which encouraged practice changes. Key words: unplanned extubation; accidental extubation; self-extubation; lean methodology; process improvement; unplanned device removal. [Respir Care 2016;61(12):1567-1572. (C) 2016 Daedalus Enterprises]
\end{abstract}

\section{Introduction}

Invasive ventilatory support is common in premature infants admitted to the NICU. A myriad of conditions

Ms Powell is affiliated with the Department of Respiratory Care, Akron Children's Hospital of the Mahoning Valley, Boardman, Ohio. Ms Gilbert is affiliated with the Center for Operations Excellence, and Ms Volsko is affiliated with the Department of Nursing Administration, Akron Children's Hospital, Akron, Ohio.

The authors have disclosed no conflicts of interest.

Correspondence: Bonnie M Powell RRT-NPS, Akron Children's Hospital, 6505 Market Street, Boardman, $\mathrm{OH}$ 44512. E-mail: bpowel12@chmca.org.

DOI: $10.4187 /$ respcare. 04540 require assisted ventilation, which, the literature reports, improves mortality in premature infants. ${ }^{1}$ However, intubation and mechanical ventilatory support are interventions that carry risk. Unplanned device removal is one of the more serious risks associated with invasive ventilation. Unplanned extubations are the fourth most common safety event that occurs in North American NICUs. ${ }^{2}$ Unplanned extubations can lead to iatrogenic injury and have the potential to contribute to serious safety events. ${ }^{3}$ Health-care organizations focus on reducing the number of preventable safety events by implementing programs or process improvement initiatives. Historically, our Level 3 B NICU experienced a high occurrence of unplanned endotracheal tube (ETT) removals. As a result, our NICU staff accepted this as an unpreventable event. 
The literature reports the short- and long-term adverse effects associated with unplanned extubation and the subsequent re-intubation. These include cardiopulmonary deterioration; ventilator-associated pneumonia; hypoxemia; hypercarbia; trauma to the larynx, pharynx, and trachea; and subglottic stenosis. ${ }^{3-5}$ Patients admitted to the NICU are at particularly high risk for an unplanned extubation, due to many factors. The process of securing an ETT and avoiding malposition is challenging in premature and micropremature infants. Specifically, the diameter of the ETT, $2.5-4.0 \mathrm{~mm}$, and the surface area of the infant's face required to affix the tube are small, and the infant's skin is fragile. Infants are often cared for in a high-humidity temperature-controlled Isolette. This warm and moist environment reduces the adhesiveness of the tape or commercial securing devices. Malpositioning can easily occur due to the extremely short length of the trachea and patient movement, which is often not restricted, since the practice in most NICUs is to limit the amount of sedation used. ${ }^{6}$ Patient movement also includes frequent repositioning and removal of the infant from the Isolette to parent arms for bonding and kangaroo care.

\section{See the Related Editorial on Page 1704}

There is currently no nationally accepted benchmark for the number of acceptable unplanned extubations in the NICU; however, in 2012, the Vermont Oxford Network suggested 2 unplanned extubations/100 intubated days should be the highest acceptable rate. ${ }^{4}$ The purpose of this study was to use lean methodology to evaluate the effect of a process improvement initiative on the unplanned extubation rate in a Level 3B NICU. We hypothesized that the use of a rapid-cycle PDSA (plan, do, study, act) initiative would reduce the unplanned extubation rate.

\section{Methods}

Baseline unplanned extubation data, including date, time, and description of event, were collected from November 1, 2012 to June 6, 2014 from the hospital safety event reporting system (Midas 2012.1.6, Midas + Solutions, Tucson, Arizona). An unplanned extubation was defined as the removal of an ETT without the order of a licensed provider. All intubated infants in the Level 3B NICU at Akron Children's Hospital Mahoning Valley were included in this study.

A voice of the customer survey was used to ascertain staff perceptions regarding unplanned extubation causes and impact on care. This voice of the customer survey assessed the staff's readiness for change and identified what the staff valued. A fundamental principle in lean methodology is to understand value. Value is defined by the downstream customer of a product or service. As an integral part of any project that uses the concepts of Lean and Six Sigma, it is important to

\section{QUICK LOOK}

\section{Current knowledge}

The literature clearly defines a myriad of the possible complications that can result from an unplanned extubation. There is very little evidence to support any one measure that improves the unplanned extubation rate.

\section{What this paper contributes to our knowledge}

Lean methodology was used to select high impact improvements to aid in the reduction of unplanned extubations. A statistically significant reduction in unplanned extubations was seen in the post-improvement phase of this study.

understand what is valued or critical to quality for our internal and external customers. We call this obtaining the "voice of the customer." 7 The anonymous and confidential survey contained 2 open-ended and 4 closed-ended questions and was distributed to a random sample of registered nurses (RNs) and respiratory therapists (RTs).

A multidisciplinary team, consisting of 4 RNs, 4 RTs, a neonatal nurse practitioner, and a neonatologist used lean methodology to draft a fishbone diagram or tool used to identify sources of defects. ${ }^{8}$ A facilitated brainstorming session as used to identify the potential cause of unplanned extubations. Figure 1 shows the fishbone diagram the team constructed to identify potential causes for unplanned extubation or our opportunities for improvement. Data for the pre-improvement phase were collected from November 2012 through May 2014. Data were collected on a random subset of the subjects $(n=19)$ identified as having an unplanned extubation event during the aforementioned data collection period. A random number generator was used to create the list, from which the subjects were selected. Information obtained from randomly selected subjects included weight, time of last ETT placement documentation, ETT location before and after the unplanned extubation, use of radiography after the unplanned extubation, use of sedation, and RN and RT staffing. Data from this data set were used to assist the team in constructing the fishbone diagram and to determine key areas for focused improvements.

An ease impact chart was used to identify the improvements that were believed to be quick fixes and perceived to make the most impact. This resulted in the recognition of 6 improvements. These improvements were implemented in 2 phases over a 2-month period using didactic and kinesthetic techniques. The 2-phase approach was used to avoid overwhelming and/or confusing the staff. Visual cues were placed on all mechanical ventilators to reinforce information provided to staff in the didactic sessions. Since staff work 12-h 


\section{Reducing UnPlanNed Extubations IN THE NICU}

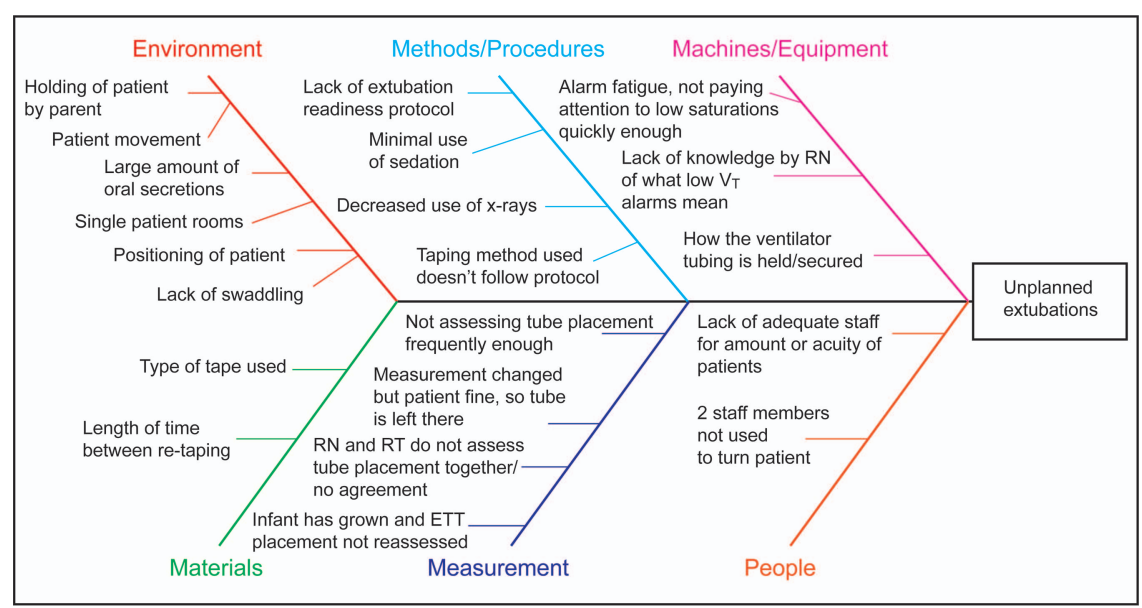

Fig. 1. Fishbone diagram of factors contributing to unplanned extubations. $\mathrm{RN}=$ registered nurse; $\mathrm{RT}=$ respiratory therapist; ETT = endotracheal tube.

shifts, this stepped approach provided time for integration of the improvements with all staff, including those who work part time and per diem. The first phase was instituted on June 14, 2014 and included the following improvements. (1) A standardized process for turning intubated infants was adopted. All infants with an ETT required the use of 2 caregivers for any activity that required turning the head. One caregiver's sole responsibility was holding the ETT at the lip to ensure that the movement did not change the ETT position. (2) Weekly assessment of ETT placement with growth was implemented. Evaluation of ETT placement and growth, or weight gain, was addressed every Monday during family-centered rounds. The process commenced with the RT presenting the date of the last chest radiograph, the location of the ETT on film, and the current position of the ETT. The licensed provider reported the infant's weight gain from the past week. Based on the aforementioned information, the clinical team discussed the need to advance the ETT. The decision to reposition the ETT was based on Neonatal Resuscitation Program Guidelines for weight and ETT placement. ${ }^{9}$ Repositioning occurred immediately after the clinical team convened a decision and reduced the use of chest radiography in the decision making process. (3) Direct caregiver communication regarding tube position was addressed. This improvement resulted in the RN and RT performing their first patient assessment simultaneously, so ETT position could be discussed and evaluated as a team.

Phase 2 commenced on July 11, 2014 and addressed the following. (1) A process for addressing ETT migration was implemented. The respiratory and nursing staffs were asked to identify and investigate any small change in the ETT position, even if there was no change in the patient's condition. (2) ETT re-securement was addressed. A standardized taping method was already in place; however,
RNs and RTs were asked to assess the integrity of the tape together. (3) Head position during radiologic studies was standardized, and RTs were required to hold for all films on intubated patients, not just those designated for ETT placement. General information on how positioning of the head during a film affects ETT movement was also provided to staff.

In the control phase of this project, all unplanned extubations triggered a chart review. The key components of care and opportunities for improvement on each case were presented in detail to the NICU multidisciplinary quality improvement committee monthly. Feedback was also provided to staff members involved in the direct care of infants with an unplanned extubation regarding areas for improvement. The Fisher exact test was used to determine differences in the number of unplanned extubations per 100 intubated days and sex differences in the pre- and post-improvement groups. $t$ tests determined differences in the patient population in the pre- and post-improvement phases. Descriptive statistics were used to report survey results. Statistical significance was established at $P<.05$.

\section{Results}

Our voice of the customer survey response rate was $68 \%$ (17 of 25) (Table 1). A random sample of subjects ( $n=19)$ during the 19-month pre-improvement phase revealed that $74 \%(n=14)$ of those needing re-intubation required the ETT to be advanced within the trachea, and $26 \%(n=5)$ of this subset were successfully maintained on noninvasive ventilation. Unplanned extubations in the post-improvement phase occurred in 7 subjects, which resulted in a statistically significant improvement in the rate of unplanned extubations $(P=.01)$ (Fig. 2). The mean age of neonates in the post-improvement group was less than in the pre-improvement group $(P=.009)$ (Table 2$)$. No 


\section{Reducing UnPlanNed Extubations IN THE NICU}

Table 1. Results of Voice of the Customer Survey

\begin{tabular}{|c|c|c|c|c|}
\hline Question & Yes, $n(\%)$ & No, $n(\%)$ & Sometimes, $n(\%)$ & Not Answered, $n(\%)$ \\
\hline Do you feel that unplanned extubations are happening too frequently? & $6(35)$ & $10(59)$ & NA & $1(6)$ \\
\hline $\begin{array}{l}\text { Do you feel that some of the unplanned extubations could be } \\
\text { prevented? }\end{array}$ & $15(88)$ & $2(12)$ & NA & NA \\
\hline $\begin{array}{l}\text { Do you feel personally responsible when an unplanned extubation } \\
\text { occurs on your patient? }\end{array}$ & $6(35)$ & $6(35)$ & $5(30)$ & NA \\
\hline $\begin{array}{l}\text { Do you feel responsible when an unplanned extubation occurs even } \\
\text { when you have taken every measure possible to prevent it? }\end{array}$ & $4(24)$ & $9(53)$ & $3(17)$ & $1(6)$ \\
\hline
\end{tabular}

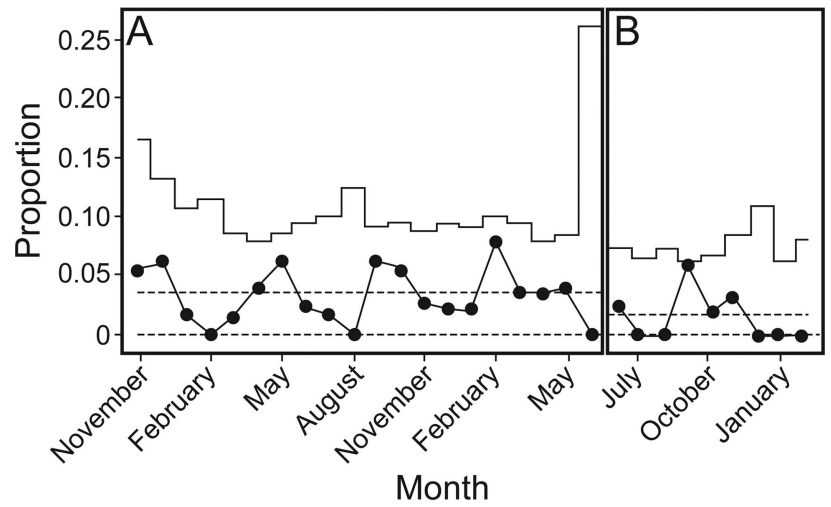

Fig. 2. Control chart displaying the number of unplanned extubations in the pre-improvement $(A)$ and post-improvement phases (B). Before improvement, there were 3.8 unplanned extubations $/ 100$ intubated days (A). After improvement, there were 2.7 unplanned extubations/100 intubated days (B). Tests were performed with unequal sample sizes. Mean $P=.02$. Dashed lines indicate upper and lower control limits.

Table 2. Comparison of Demographics for the Subset of Subjects With Unplanned Extubations Included in the Preimprovement Period and Post-improvement Phase

\begin{tabular}{lccc}
\hline \hline & Pre-improvement Post-improvement & $P$ \\
\hline Subjects, $n$ & 19 & 20 & \\
Weight at time of unplanned & & & \\
$\quad$ extubation, g & $1,423.7$ & $1,314.3$ & .64 \\
$\quad$ Mean + SD & 613.7 & 813.2 & \\
$\quad$ Range & & & \\
Age, days & 45.5 & 26.8 & .009 \\
$\quad$ Mean + SD & 22.1 & 22.1 & \\
$\quad$ Range & & & \\
Sex, $n(\%)$ & $13(68)$ & $8(40)$ & .08 \\
$\quad$ Male & $6(32)$ & $12(60)$ & \\
$\quad$ Female & &
\end{tabular}

statistically significant differences in weight or sex were found (Table 2). There was a concomitant decrease in the number of intubated days between the pre- and post-improvement groups $(P<.001)$ (Fig. 3).

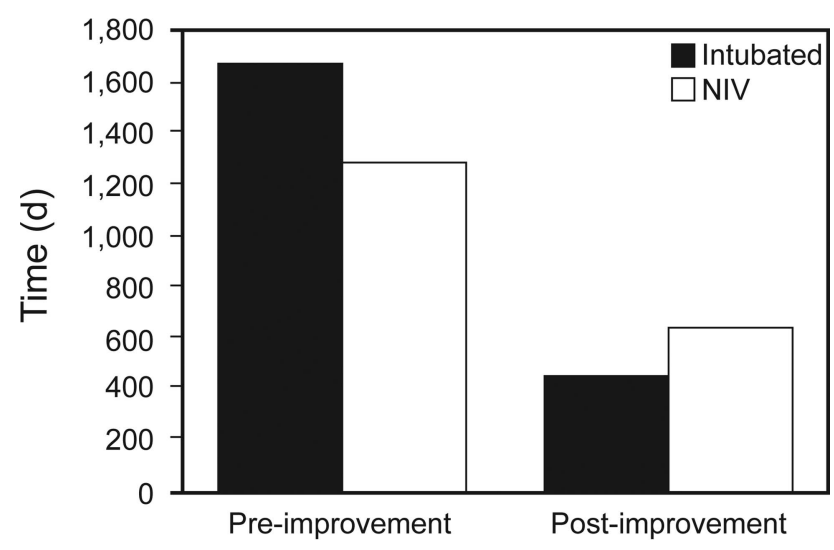

Fig. 3. Comparison of invasive and noninvasive ventilator days pre- and post-improvement. This improvement was statistically significant, $P<.001$.

\section{Discussion}

This project was initiated with a voice of the customer survey to assess cultural readiness for change. During the define phase of this process improvement, the NICU moved from an open bay design to private rooms. The walking distance between patients and the size of the new singlepatient room NICU (4 times the square footage of the open unit) required the staff to adjust to new workflows. This transition presented some initial difficulty, especially with the simultaneous implementation of some of the improvements. We anticipated that the 2 caregivers to turn model would be the most difficult to implement, but staff were able to adapt. Survey results demonstrated to our process improvement team that the culture did not support the perception that unplanned extubations were always an unpreventable event. The majority of respiratory and nursing staff reported that at least some of the unplanned extubations were preventable. The free text portion of the survey provided a number of comments from staff stating how the events affected them on a personal and professional level. Overall, the results displayed a desire by staff to improve the safety of the care provided to their patients. 
During this define and measure portion of our project, staff voiced concerns that they perceived unplanned extubations occurred more frequently with larger infants and when staffing levels were not appropriate. Data from the analysis phase showed no statistical difference in patient weight, a surrogate for size, which did not support their perceptions. Additionally, staffing was at the planned level when unplanned extubations occurred. When this information was relayed to staff, the educators reviewed the physiological and environmental factors contributing to unplanned extubations that the improvements targeted. These factors were similar to those identified in the literature and were aligned with countermeasures implemented during our improve phase of the PDSA project. In a comprehensive review, Silva et $\mathrm{al}^{2}$ reported that risk factors for unplanned extubations included restlessness/agitation (13-89\%), poorly fixated ETT $(8.5-31 \%)$, ETT manipulation (17-30\%), and performance of bedside procedures $(27.5-51 \%)$. Veldman et $\mathrm{al}^{10}$ reported unplanned extubations occurred most frequently when fixation of the ETT was difficult, handling of the infant by nursing staff or during care or with active infants in whom dislocation of the ETT occurred without external manipulations.

Lean and Six Sigma principles were used in this process improvement initiative to engage those who perform beside care to identify and correct defects. An ease impact chart allowed staff to identify the quick wins or opportunities that would make an impact without significant investment in resources and time. Similar to reports in the literature, ${ }^{11}$ the use of this lean tool further engaged participants in the change.

The data collection period was significantly longer for the pre-improvement group. The acuity of the NICU influenced the number of intubated days. There were periods where admissions of premature infants requiring intubation and mechanical ventilation were low. Rather than using raw numbers, we controlled for this variance by comparing the rate of unplanned extubations using the formula: number of unplanned extubations/100 intubated days. Therefore, the proportion of intubated days was identical for both the pre- and the post-improvement groups. The difference in the data collection periods was attributed to the variance in volume and patient acuity in our NICU.

Two improvements evidenced the most change: ETT assessment for growth and addressing ETT migration. Routine chest radiographs to assess ETT placement are no longer a practice in our NICU. The literature reports risks associated with radiation exposure in the neonatal period, ${ }^{12-14}$ which influenced our practice in the NICU. Rather than obtain a chest radiograph daily to assess ETT position, chest radiographs are only obtained when a clinical indication, such as verification of central line placement or clinical deterioration, exists. Since chest radiography was conducted only when needed, the improvement team recognized the value of standardizing practice and position- ing the infant to also facilitate ETT evaluation. Changes in practice, specifically RTs holding the head of the intubated infant midline for any portable radiologic study, were implemented. This improvement enabled the team to evaluate ETT position in addition to using the image to evaluate the condition for which it was ordered.

During the analysis phase, we found that the relationship between patient growth and the need to reposition the ETT was not being routinely addressed by the clinical team. Knowledge gaps may have contributed to the inability of the nursing and respiratory staff to appreciate the importance of assessing growth and its relationship to ETT malposition. Our focus on discussing ETT position in relation to growth weekly during patient family-centered rounds resulted in improved awareness of this concept and a structured process to identify and correct ETT malposition.

The timing of patient assessments, specifically simultaneous RT and RN assessments, allowed ETT securement and position to be evaluated. This bedside team investigated and addressed small changes, often only one quarter $\mathrm{cm}$, in the movement of the ETT and problems with the adhesiveness of the tape. Collaboration between RTs and RNs not only identified potential causes for an unplanned extubation but provided sufficient staff at the bedside to correct the defect. Through a process of coordinating care, we were able to avoid unintentional movement of the ETT.

Data collected in the pre-improvement phase showed that a significant number of subjects with unplanned extubations were not re-intubated. During this phase of the study, there were also a higher number of intubated days and a lower number of noninvasive days. When subject demographics were analyzed, no appreciable differences in the pre- and post-improvement groups were detected. However, the number of orders, in the electronic health record, to wean from invasive support increased in the post-improvement group. We suspect that in the post-improvement phase, there was an increased use of noninvasive ventilation, which was influenced by earlier extubation.

In our current practice, we investigate all of our unplanned extubations and provide closed-loop feedback to staff. Each time an unplanned extubation occurs, a thorough chart review is conducted, and information obtained is reviewed with staff. Staff identifies the defects as well as improvements to help us to prevent further unplanned extubations. The review is done in a non-punitive manner. A cumulative report of unplanned extubations are reviewed at the interdisciplinary performance improvement meetings monthly. Continuous review and closed-loop feedback to staff identify deviations from standard practice and increase staff awareness of the importance of adhering to the aforementioned practice changes. Reviewing our progress toward our goal of 1 unplanned extubation/ 100 intubated days also helps to change the culture in the NICU to a high-reliability learning culture and one that embraces opportunities to enhance patient safety. 


\section{Reducing UnPlanNed Extubations IN THE NICU}

The use of a PDSA process allowed our NICU care team to implement better practices or process changes. Similar to published reports in the literature, ${ }^{3,4,15}$ we were able to reduce unplanned extubations in our NICU through staff education and the implementation of standard practices of care.

There were a number of limitations to this study. The data were retrospectively collected in the pre-improvement phase, and the cause(s) leading to unplanned extubation could not always be identified. There may have been more than one contributing factor to the unplanned extubation, which, due to data collection limitations, could not be identified. For example, a note in the patient medical record might identify that formula was seen in the ETT, which prompted extubation and evaluation for the need to re-intubate or support the infant on either noninvasive ventilation or oxygen therapy. However, this patient may have been turned by a single caregiver, without assistance before the event, but never documented. Rather than reviewing all unplanned extubations, a random sample of unplanned extubation events were selected for this study. This presents a potential limitation in that all possible opportunities and evaluation of process improvements could not be identified.

As a result, our data collection tool and process for reviewing unplanned extubation events have changed. Our tool is very prescriptive and is completed by all members of the direct care team involved in the care of the child at the time of the unplanned extubation. Multidisciplinary review of the event and completion of our unplanned extubation tool must now occur within $12 \mathrm{~h}$ of the event.

Reliance on staff self-reporting unplanned extubation occurrences in our safety reporting system was also a limitation. To gather reliable data, redundant processes are needed to minimize the propensity of not accurately capturing all events. In addition to our data collection tool, described above, we are currently in the process of revising our electronic health record to capture unplanned extubation events.

\section{Conclusions}

Staff underestimated the prevalence of unplanned extubations but recognized the need for improvement. Personal accountability enhanced engagement with the improvement process. Rapidcycle PDSA improved patient safety by significantly reducing the unplanned extubation rate in the NICU. The concomitant decrease in the number of intubated days may have been a by-product of the post-improvement phase improvements, which encouraged practice changes. Improvement work takes a great deal of diligence and commitment to institute and may be even more difficult to maintain over time.

\section{ACKNOWLEDGMENTS}

We thank all of the direct care providers from the Akron Children's Hospital Mahoning Valley NICU who participated in this quality initiative.

\section{REFERENCES}

1. Carlo WA. Gentle ventilation: the new evidence from the SUPPORT, COIN, VON, CURPAP, Columbian Network, and Neocosur Network Trials. Early Hum Dev 2012;88(Suppl 2):S81-S83.

2. Silva PS, Reis ME, Aguiar VE, Fonseca MC. Unplanned extubation in the neonatal ICU: a systematic review, critical appraisal, and evidencebased recommendations. Respir Care 2013;58(7):1237-1245.

3. Barber J. Unplanned extubation in the NICU. J Obstet Gynecol Neonatal Nurs 2013;42(2):233-238.

4. Merkel L, Beers K, Lewis MM, Stauffer J, Mujsce DJ, Kresch MJ. Reducing unplanned extubations in the NICU. Pediatrics 2014;133(5): e1367-e1372.

5. Loughead JL, Brennan RA, DeJuilio P, Camposeo V, Wengert J, Cooke D. Reducing accidental extubation in neonates. Jt Comm J Qual Patient Saf 2008;34(3):164-170, 125.

6. Little LA, Koenig JC Jr, Newth CJ. Factors affecting accidental extubations in neonatal and pediatric intensive care patients. Crit Care Med 1990;18(2):163-165.

7. Womack, JP, Jones, DT. Lean thinking: banish waste and create wealth in your corporation, 2nd edition. New York: Free Press; 2003:189-218.

8. Tague NR. The quality toolbox, 2nd edition. Milwaukee, Wisconsin: American Society for Quality; 2005:247-249.

9. Kattwinkel J, editor. Textbook of neonatal resuscitation, 6th edition. Dallas: American Heart Association and American Academy of Pediatrics; 2011:182.

10. Veldman A. Trautschold T, Weiss K, Fischer D, Bauer K. Characteristics and outcome of unplanned extubation in ventilated preterm and term newborns on a neonatal intensive care unit. Paediatr Anaesth 2006;16(9):968-973.

11. Kimsey DB. Lean methodology in health care. AORN J 2010;92(1): 53-60.

12. Armpilia CI, Fife IA, Croasdale PL. Radiation dose quantities and risk in neonates in a special care baby unit. Br J Radiol 2002; 75(895):590-595.

13. Hammer GP, Seidenbusch MC, Schneider K, Regulla DF, Zeeb H, Spix C, Blettner M. A cohort study of childhood cancer incidence after postnatal diagnostic x-ray exposure. Radiat Res 2009;171(4):504-512.

14. Hammer GP, Seidenbusch MC, Regulla DF, Spix C, Zeeb H, Schneider K, Blettner M. Childhood cancer risk from conventional radiographic examinations for selected referral criteria: results from a large cohort study. AJR 2011;197(1):217-223.

15. Rachman BR, Mink RB. A prospective observational quality improvement study of the sustained effects of a program to reduce unplanned extubations in a pediatric intensive care unit. Paediatr Anaesth 2013;23(7):614-620.

This article is approved for Continuing Respiratory Care Education credit. For information and to obtain your CRCE

(free to AARC members) visit

www.rcjournal.com

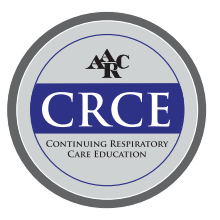

